\title{
Reference values for amino acids and acylcarnitines in peripheral blood in Quarter horses and American Miniature horses
}

\author{
Irám Pablo Rodríguez-Sánchez ${ }^{1 *}$, Víctor Manuel Treviño-Alvarado² , María del Rosario Torres-Sepúlveda , \\ Liliana Aracely López-Saldaña ${ }^{3}$, Gustavo Ponce-García', Graciela Areli López-Uriarte? \\ María del Consuelo Ruiz-Herrera' ${ }^{1}$, Diana Elisa Zamora-Ávila ${ }^{3}$, Jesús Zacarías Villarreal-Pérez ${ }^{5}$, \\ Guillermo Dávalos-Aranda ${ }^{3}$ and Laura Elia Martínez-de-Villarreal ${ }^{1}$
}

\begin{abstract}
Background: Free amino acids and acylcarnitines circulating in the blood can be used for diagnosis for metabolic illness and imbalances. To date, the normal reference ranges of amino acids and acylcarnitines in horse peripheral blood have not been established. In this study, the concentrations of 12 amino acids and 26 acylcarnitines were determined by tandem mass spectrometry in complete blood from 100 healthy horses (50 Quarter horses (QH) $[23$ males and 27 females] and 50 American Miniature horses (AMH) [15 males and 35 females]) with no signs of metabolic disease. The means and standard deviations were determined and data statistically analyzed.

Findings: Concentrations of short, medium, and long chain acylcarnitines were significantly higher in male AMH than in male QH. The concentrations of the amino acids alanine, arginine, glycine, proline (glycogenic), and leucine (ketogenic) were higher in the $\mathrm{QH}$ than in the $\mathrm{AMH}$. Female $\mathrm{AMH}$ had higher concentrations of propionylcarnitine, leucine, proline, arginine, and ornithine than female $\mathrm{QH}$.
\end{abstract}

Conclusions: Normal reference ranges of amino acids and acylcarnitines were established for AMH and QH. Significant differences were found in concentration of these compounds between breeds and gender.

Keywords: Amino acid, Acylcarnitine, Metabolic profile, Miniature horse, Quarter horse, Tandem mass spectrometry

\section{Findings}

Analysis of free amino acids and acylcarnitines circulating in blood is a powerful diagnostic tool for metabolic illnesses and imbalances [1]; conditions that have an economic impact in the equine sector [2]. Reference ranges for these compounds have been established for obese rats, adult canines and even for plants by tandem mass spectrometry (MS/MS) [3-5] and atypical myopathies

\footnotetext{
*Correspondence: iramrodriguez@gmail.com

1 Departamento de Genética, Hospital Universitario "Dr. José Eleuterio González", Universidad Autónoma de Nuevo León, Monterrey, Nuevo León, Mexico

Full list of author information is available at the end of the article
}

have been identified in horses (Equus caballus) using this technology [6]. Metabolic disorders are common in horses, and can be categorized as disorders of carbohydrate metabolism [7], lipid disorders [8], purine disorders [9], and primary disorders of electrolytic flux [10]. Diet [11] and metabolic disorders are important factors affecting amino acid and acylcarnitine concentrations in horses [12].

We hypothesized that concentrations of amino acids and acylcarnitines in the blood are different between Quarter horses $(\mathrm{QH})$ and American Miniature horses $(\mathrm{AMH})$. This study reports the concentrations of these compounds in peripheral blood of normal horse of both 
gender and establishes normal reference ranges for these breeds.

The research protocol was approved by the Institutional Committee for the Act \# 2 (Approbation code FMVZ-CBA-002). A total of 100 clinically normal horses [50 QH (23 males and 27 females) and $50 \mathrm{AMH}$ (15 males and 35 females)] from several stud farms and riding stables in the cities of Monterrey, Nuevo León and Cuatrociénegas, Coahuila, northeastern Mexico were selected. The horses were fed twice a day with dry forage ( $2.2 \%$ of their body weight) and commercial cereals, molasses, oilseeds, vitamins and minerals ( $0.5 \%$ of their body weight), and water ad libitum. The horses were used in racing, rodeos, or kept as pets. Age and weight ranges are shown in detail in Additional file 1.

Complete blood was collected 3-5 h after first food ingestion in the morning from the jugular vein using a vacutainer tube with no anticoagulant and a $21 \mathrm{G} \times 1.5^{\prime \prime}$ size needle; posteriorly one drop of blood was placed on each of five filter paper circles (S\&S 903, Whatman, Little Chalfont, UK) using a pipette. The filter papers were then dried for $3 \mathrm{~h}$ at room temperature avoiding exposure to sunlight.
Each sample was analyzed for 12 amino acids and 26 acylcarnitines (Fig. 1). A $3.2 \mathrm{~mm}$ circle was obtained using a Wallac DBS Puncher (PerkinElmer, Waltham, MA, USA) from the dry filter paper. NeoBase non-derivatized MS/MS kit (PerkinElmer) was used to obtain the metabolites of interest, following the manufacturer's instructions. A solution included in the kit containing internal standards labeled with stable isotopes was used for quantification of the metabolites of interest. The prepared samples were analyzed by MS/MS (API 2000, ABSciex, Framingham, MA, USA) coupled to a micropump and an autosampler (Series 2000, Perkin Elmer). Sample analysis was performed with multiple reaction monitoring using Analyst 1.6.2 Software (ABSciex) and the NeoBase database. The results were interpreted using Analyst 1.6.2 Software.

Statistical analysis was performed using R (http://www. cran.r-project.org/). To normalize the raw measurements, we set the sum of all values within a sample to 1 . This was achieved by dividing each value by the sum of all values per sample. These normalized values were then converted to logarithms to handle extreme values. A $t$ test was used to determine statistically significant differences between groups. Corrections of q-values were made for
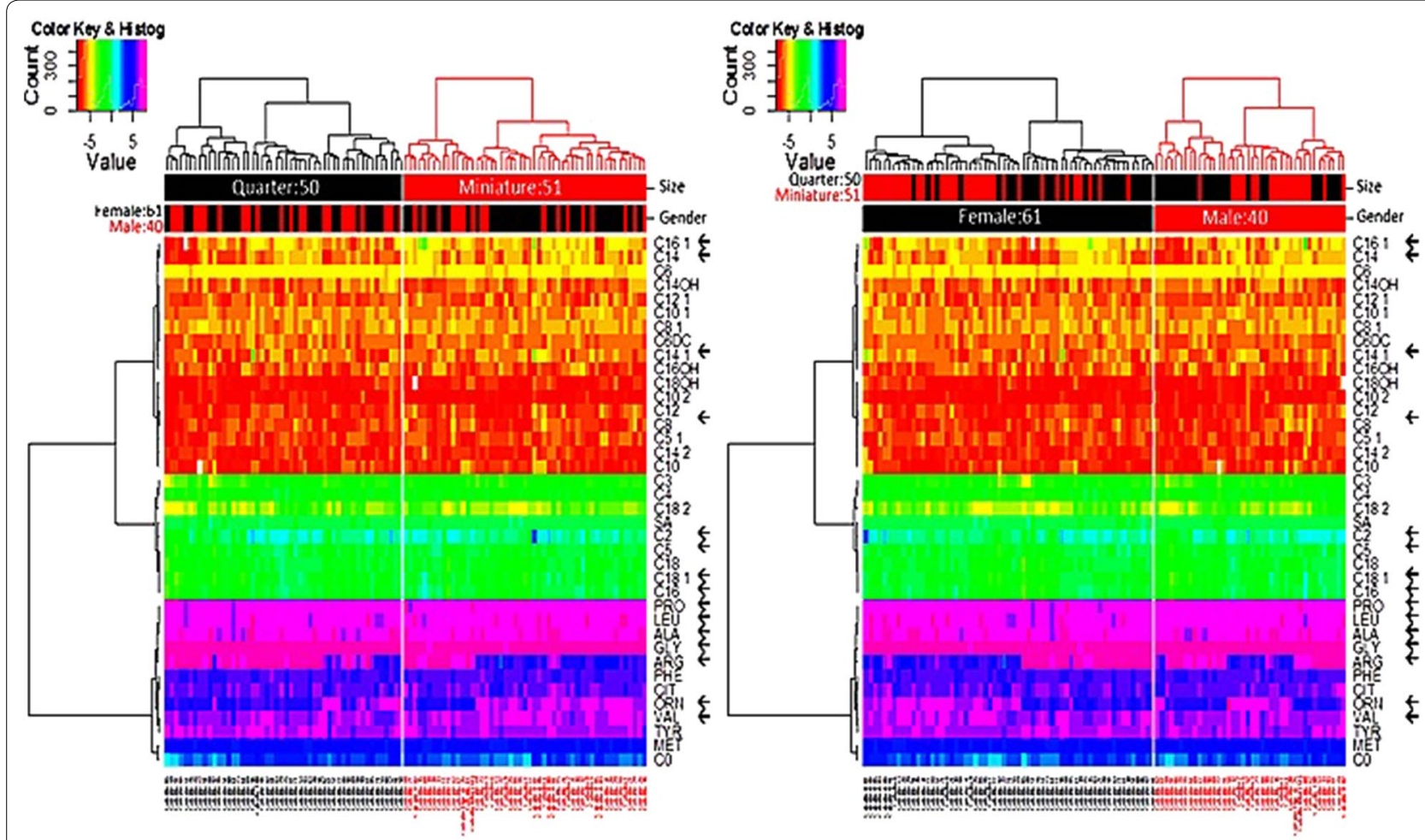

Fig. 1 Heat map representing concentrations of metabolites analyzed. Left panel shows data order by size. Right panel shows concentrations by gender. Horses are shown in columns and metabolites in rows, which are grouped by hierarchical clustering for clarity. Color code is shown. Arrows highlight significant changes 
multiple tests using a false discovery rate approach and $P \leq 0.05$ were considered as significant. For correlation analyses, absolute Pearson correlations $\geq 0.7$ were used.

Normal reference ranges were established for 12 amino acids and 26 acylcarnitines in blood of AMH and QH. Mean values, standard deviation, maximum and minimum values for each metabolite are provided in Additional file 2 for each breed and gender. Among the 38 metabolites (Fig. 1), the concentrations of 16 were significantly different between the horse breeds (indicated by * Table 1).

Comparisons by gender between breeds (female quarters vs female miniatures, and the same for males) were statistically significant for both males and females (Table 1; Fig. 1), but not within the same breed. For male horses, concentrations of C2, C3, C5, C8, C14, C14:1, $\mathrm{C}: 16$ and $\mathrm{C} 16: 1$ were significantly higher in $\mathrm{AMH}$ than in QH. Only C18:1 was higher in male QH than in male $\mathrm{AMH}$. The concentrations of the amino acids Ala, Arg, Gly, Pro and Leu were significantly higher in $\mathrm{QH}$ than in $\mathrm{AMH}$, while the concentrations of Val was higher in $\mathrm{AMH}$ than in $\mathrm{QH}$. For the female horses, the concentrations of C3, Leu, Pro and Arg were significantly higher in $\mathrm{AMH}$ than in $\mathrm{QH}$. Inversely, Val and Orn were higher in $\mathrm{QH}$ than in AMH (Table 1; Fig. 1).

In the present study, horse breeds showed differences in acylcarnitine concentrations, which could be caused by variation in daily physical activity, individual genetics, or environmental factors. Diet is a factor that affects blood concentrations of amino acids, hormones, proteins, and other circulating compounds [11]. In the present study, horses were fed the same ration based on their individual weight, so the effects of diet on the obtained values is considered minimal. QH showed higher Leu concentrations than $\mathrm{AMH}$ and conversely Val concentrations were higher in male $\mathrm{AMH}$ than in male $\mathrm{QH}$. These differences may reflect metabolic differences between breeds. Analysis of samples from other breeds may clarify this. If the differences depend on breed, this factor should be taken into account in the interpretation of the results for the possible diagnosis of metabolic disorders.

In conclusion, normal reference ranges were established for amino acids and acylcarnitines in $\mathrm{QH}$ and $\mathrm{AMH}$. The established ranges could be used to diagnose metabolic abnormalities that affect the performance of horses of high sporting and economic value. Positive results can be followed up with more specific testing for confirmation of the diagnosis.
Table 1 Overview of analyzed metabolites in American Miniature horses (AMH) $(n=50)$ and Quarter horses (QH) (n= 50)

\begin{tabular}{|c|c|c|c|c|}
\hline \multirow[t]{2}{*}{ Metabolites } & \multicolumn{4}{|c|}{ Compared groups } \\
\hline & $\mathrm{QH} / \mathrm{QH}_{0}$ & QHO/AMHO & $\mathrm{QH}_{\delta} / \mathrm{AMH}_{\circlearrowleft}$ & AMHO/AMH ${ }^{\star}$ \\
\hline $\mathrm{CO}$ & 0.87 & 0.44 & 0.73 & 0.93 \\
\hline $\mathrm{C} 2$ & 0.61 & 0.44 & $0.03^{*}$ & 0.86 \\
\hline C4 & 0.87 & 0.14 & 0.10 & 0.86 \\
\hline C6 & 0.61 & 0.44 & 0.83 & 0.96 \\
\hline C6DC & 0.29 & 0.81 & 0.06 & 0.86 \\
\hline $\mathrm{C} 8$ & 0.52 & 0.81 & $0.01^{*}$ & 0.86 \\
\hline C8.1 & 0.99 & 0.07 & 0.35 & 0.86 \\
\hline $\mathrm{C} 10$ & 0.61 & 0.16 & 0.58 & 0.93 \\
\hline C10.1 & 0.80 & 0.45 & 0.14 & 0.86 \\
\hline C10.2 & 0.52 & 0.81 & 0.19 & 0.93 \\
\hline $\mathrm{C} 12$ & 0.36 & 0.81 & 0.06 & 0.86 \\
\hline C12.1 & 0.77 & 0.41 & 0.22 & 0.86 \\
\hline $\mathrm{C} 14$ & 0.80 & 0.06 & $0.01^{*}$ & 0.86 \\
\hline C14.1 & 0.61 & 0.15 & $0.04^{*}$ & 0.96 \\
\hline C14.2 & 0.61 & 0.81 & 0.14 & 0.86 \\
\hline $\mathrm{C} 14 \mathrm{OH}$ & 0.61 & 0.81 & 0.35 & 0.93 \\
\hline $\mathrm{C} 16$ & 0.80 & 0.15 & $0.01^{*}$ & 0.86 \\
\hline C16.1 & 0.61 & 0.20 & $0.01^{*}$ & 0.86 \\
\hline $\mathrm{C} 16 \mathrm{OH}$ & 0.90 & 0.98 & 0.74 & 0.86 \\
\hline $\mathrm{C} 18$ & 0.61 & 0.81 & 0.20 & 0.86 \\
\hline C18.1 & 0.61 & 0.29 & $0.01^{*}$ & 0.86 \\
\hline C18.2 & 0.61 & 0.98 & 0.38 & 0.93 \\
\hline $\mathrm{C} 18 \mathrm{OH}$ & 0.61 & 0.61 & 0.10 & 0.93 \\
\hline C5 & 0.61 & 0.41 & $0.03^{*}$ & 0.86 \\
\hline C5.1 & 0.61 & 0.41 & 0.12 & 0.86 \\
\hline C3 & 0.87 & $0.04^{*}$ & $0.03^{*}$ & 0.86 \\
\hline ALA & 0.61 & 0.06 & $0.01^{*}$ & 0.93 \\
\hline ARG & 0.89 & $0.00^{*}$ & $0.03^{*}$ & 0.86 \\
\hline CIT & 0.87 & 0.45 & 0.07 & 0.86 \\
\hline GLY & 0.36 & 0.45 & $0.03^{*}$ & 0.93 \\
\hline LEU & 0.61 & $0.00^{*}$ & $0.00^{*}$ & 0.86 \\
\hline MET & 0.89 & 0.43 & 0.78 & 0.86 \\
\hline ORN & 0.97 & $0.01^{*}$ & 0.15 & 0.86 \\
\hline PHE & 0.80 & 0.28 & 0.14 & 0.93 \\
\hline PRO & 0.80 & $0.00^{*}$ & $0.01^{*}$ & 0.86 \\
\hline SA & 0.61 & 0.23 & 0.22 & 0.86 \\
\hline TYR & 0.61 & 0.11 & 0.38 & 0.86 \\
\hline VAL & 0.87 & $0.00^{*}$ & $0.04^{*}$ & 0.86 \\
\hline
\end{tabular}

The data were statistically compared according to breed (AMH vs. QH) and gender ( $\sigma^{\star}$ vs. ㅇ). See Abbreviations for metabolite abbreviations

* Represents a $P$ value of $P \leq 0.05$ 


\section{Additional files}

Additional file 1: Phenotypic characteristics of the horses of both breeds. Includes phenotypical characteristics (breed, sex, age, weight, height) and the relationship between individual of the group.

Additional file 2: Statistic data. Includes mean, standard deviation, minimum and maximum ranges for the metabolites analyzed.

\section{Abbreviations}

Ala: alanine; $\mathrm{AMH}$ : American Miniature horses; Arg: arginine; CO: free carnitine; C4: butyrylcarnitine; C5: 1: tiglylcarnitine; C6: hexanoylcarnitine; C6DC: adipylcarnitine; C8: 1: octenoylcarnitine; C10: decanoyl carnitine; C10: 1: decenoyl carnitine; C10: 2: decadienoyl carnitine; C12: dodecanoyl carnitine; C12: 1 dodecenoyl carnitine; C14: 2: tetradecadienoyl carnitine; C14OH: 3-hydroxytetradecanoyl carnitine; $\mathrm{C} 16 \mathrm{OH}: 3$ hydroxy-hexadecanoyl carnitine; $\mathrm{C} 16: 1 \mathrm{OH}$ : 3-hydroxy-hexadecenoyl carnitine; C18: octadecanoyl carnitine (estearoy carnitine); C18: 2: octadecadienoyl carnitine (linoleyl carnitine); $\mathrm{C} 180 \mathrm{H}$ : 3-hydroxy-octadecanoyl carnitine; C18: 1OH: 3-hydroxy-octadecenoyl carnitine; Cit: citrulline; Gly: glycine; Leu: leucine; Met: methionine; Orn: ornithine; Phe: phenylalanine; Pro: proline; QH: Quarter horses; SA: succinylacetone; Tyr: tyrosine; Val: valine.

\section{Authors' contributions}

IPRS, GDA, and LEMV designed the study. MCRH and MRTS carried out the main laboratory work. VMTA and GPG aided in analysis of results and carried out bioinformatic work. IPRS and LALS collected biological samples. GDA LALS and DEZA collected physical data. GALU, JZVP and LEMV interpreted the results. All authors read and approved the final manuscript.

\section{Author details}

1 Departamento de Genética, Hospital Universitario "Dr. José Eleuterio González", Universidad Autónoma de Nuevo León, Monterrey, Nuevo León, Mexico. ${ }^{2}$ Tecnológico de Monterrey Cátedra de Bioinformática, Campus Monterrey, Monterrey, Nuevo León, Mexico. ${ }^{3}$ Departamento de Genética, Facultad de Medicina Veterinaria y Zootecnia, Universidad Autónoma de Nuevo León, Monterrey, Nuevo León, Mexico. ${ }^{4}$ Departamento de Zoología de invertebrados, Facultad de Ciencias Biológicas, Universidad Autónoma de Nuevo León, Monterrey, Nuevo León, Mexico. ${ }^{5}$ Servicio de Endocrinología, Hospital Universitario "Dr. José Eleuterio González", Universidad Autónoma de Nuevo León, Monterrey, Nuevo León, Mexico.

\section{Acknowledgements}

The authors gratefully acknowledge the critical reading of the manuscript by Sergio Lozano-Rodriguez, MD.

\section{Compliance with ethical guidelines}

\section{Competing interests}

The authors declare that they have no competing interests.

Received: 24 February 2015 Accepted: 14 September 2015

Published online: 29 September 2015

\section{References}

1. Indiveri C, lacobazzi V, Tonazzi A, Giangregorio N, Infantino V, Convertini P, et al. The mitochondrial carnitine/acylcarnitine carrier: function, structure and physiopathology. Mol Aspects Med. 2011;32:223-33.

2. Westermann CM. Assessment of (patho)physiologic alterations in equine muscle metabolism. Utrecht: Utrecht University; 2008.

3. She $\mathrm{P}$, Olson $\mathrm{KC}$, Kadota $Y$, Inukai A, Shimomura $Y$, Hoppel $\mathrm{CL}$, et al. Leucine and protein metabolism in obese Zucker rats. PLoS ONE. 2013;8:e59443.

4. Osorio JHU-VL. Measurement of blood acylcarnitines in adult canines using tandem mass spectrometry. Vetzootec. 2007;1:24-8.

5. Bourdin B, Adenier H, Perrin Y. Carnitine is associated with fatty acid metabolism in plants. Plant Physiol Biochem. 2007;45:926-31.

6. Sas AM, van der Kolk JH, Dank M, Westermann CM. Atypical myopathy: a review and description of the outbreak in the Netherlands during autumn 2009 and spring 2010. Tijdschr Diergeneeskd. 2012;137:514-21.

7. Jeffcott LB, Field JR. Current concepts of hyperlipaemia in horses and ponies. Vet Rec. 1985:116:461-6.

8. Hc M. Equine hyperlipidaemias. Vet Clin North Am Equine Pract. 2011;27:59-72.

9. McGuire TC, Pollara B, Moore JJ, Poppie MJ. Evaluation of adenosine deaminase and other purine salvage pathway enzymes in horses with combined immunodeficiency. Infect Immun. 1976:13:995-7.

10. Westermann CM, Dorland L, Wijnberg ID, van der Kolk JH. Equine metabolic myopathies with emphasis on the diagnostic approach Comparison with human myopathies A review. Vet Q. 2007;29:42-59.

11. DePew CL, Thompson DL, Fernandez JM, Sticker LS, Burleigh DW. Changes in concentrations of hormones, metabolites, and amino acids in plasma of adult horses relative to overnight feed deprivation followed by a pellet-hay meal fed at noon. J Anim Sci. 1994;72:1530-9.

12. Ralston SL. Evidence-based equine nutrition. Vet Clin North Am Equine Pract. 2007:23:365-84

\section{Submit your next manuscript to BioMed Central and take full advantage of:}

- Convenient online submission

- Thorough peer review

- No space constraints or color figure charges

- Immediate publication on acceptance

- Inclusion in PubMed, CAS, Scopus and Google Scholar

- Research which is freely available for redistribution 